\title{
Idosos atendidos em um Serviço de Urgência e Emergência Psiquiátrica
}

\author{
Mayara Giuli dos Santos Cordeiro \\ (D) https://orcid.org/0000-0002-8011-9268 \\ Márcia Aparecida Padovan Otani² \\ (D) https://orcid.org/0000-0001-9540-4996 \\ Flávia Cristina Goulart ${ }^{2}$ \\ (D) https://orcid.org/0000-0003-0338-3214 \\ Osni Lazaro Pinheiro² \\ (D) https://orcid.org/0000-0002-6533-0428 \\ Maria José Sanches Marin ${ }^{2}$ \\ (D) https://orcid.org/0000-0001-6210-6941 \\ Carlos Alberto Lazarini ${ }^{2}$ \\ (D) https://orcid.org/0000-0003-3010-4436
}

${ }^{1}$ Santa Casa de Misericórdia, Centro Cirúrgico, Presidente Prudente, SP, Brasil.

${ }^{2}$ Faculdade de Medicina de Marília, Marília, SP, Brasil.
Objetivo: caracterizar os idosos atendidos em um Serviço de Urgência e Emergência Psiquiátrica quanto ao perfil sociodemográfico, diagnóstico psiquiátrico, prescrição de medicamentos e conduta médica e avaliar suas associações com o sexo. Método: estudo transversal, quantitativo, em que os dados foram obtidos a partir dos prontuários de pacientes atendidos no referido serviço, entre julho/2015 e junho/2016. A variável independente foi o sexo e as dependentes foram: idade; procedência; informante; tratamento psiquiátrico anterior; diagnóstico; medicamentos prescritos e conduta. Resultados: nos 152 prontuários analisados, observam-se idade prevalente entre 60 a 69 anos, maioria de mulheres, procedência do município local e comparecimento com acompanhante. Os diagnósticos prevalentes são a esquizofrenia e os transtornos mentais e comportamentais decorrentes do uso de substâncias psicoativas. As medicações prescritas com maior frequência são da classe dos antipsicóticos e anti-histamínicos, seguidas dos ansiolíticos/sedativos. O critério de Beers considera a prescrição de medicamentos do grupo dos benzodiazepínicos, antipsicóticos e anti-histamínicos inapropriada para idosos. A principal conduta foi a alta, sem encaminhamento a outro serviço. Conclusão: embora haja incentivo do Ministério da Saúde, com a criação de novas políticas de saúde mental, muitos profissionais mantêm o modelo de atendimento baseado na queixa e conduta, dificultando a reabilitação psicossocial dos pacientes.

Descritores: Idosos; Serviços de Emergência Psiquiátrica; Medicamentos; Registros Médicos.

\section{Como citar este artigo}

Cordeiro MGS, Otani MAP, Goulart FC, Pinheiro OL, Marin MJS, Lazarini CA. Elderly people served in a Psychiatric Emergency and Urgency Service. SMAD, Rev Eletrônica Saúde Mental Álcool Drog. 2021 jan.- mar.;17(1):39-47. doi: https://dx.doi.org/10.11606/issn.1806-6976.smad.2021.158278 


\section{Elderly people served in a Psychiatric Emergency and Urgency Service}

Objective: characterize the elderly treated in a Psychiatric Emergency and Urgency Service regarding their sociodemographic profile, psychiatric diagnosis, prescription of medication and medical conduct and evaluate their associations with sex. Method: a cross-sectional, quantitative study in which data was obtained from the medical records of patients seen in the referred service, between July/2015 and June/2016. The independent variable was gender and dependents were: age; origin; informant; previous psychiatric treatment; diagnosis; prescribed drugs and conduct. Results: in the 152 medical records analyzed, the prevailing age is between 60 and 69 years old, most of them women, coming from the local municipality and attending with a companion. The prevalent diagnoses are schizophrenia and mental and behavioral disorders resulting from the use of psychoactive substances. The most frequently prescribed medications are in the class of antipsychotics and antihistamines, followed by anxiolytics/sedatives. Beers' criteria consider the prescription of benzodiazepine, antipsychotic and antihistamine medications inappropriate for the elderly. The main conduct was discharge, without referral to another service. Conclusion: although there is encouragement from the Ministry of Health, with the creation of new mental health policies, many professionals maintain the model of care based on complaint and conduct, hindering the psychosocial rehabilitation of patients.

Descriptors: Elderly; Psychiatric Emergency Services; Medicines Medical Records.

\section{Asistencia de personas de edad avanzada en un servicio psiquiátrico de emergencia}

Objetivo: caracterizar a los ancianos atendidos en un servicio psiquiátrico de emergencia y urgencia en relación con el perfil sociodemográfico, el diagnóstico psiquiátrico, la prescripción de medicamentos y la conducta médica; y evaluar sus asociaciones con el sexo. Método: un estudio cuantitativo y transversal en el que se obtuvieron datos de los registros médicos de los pacientes tratados en el citado servicio, del 2015 al 2016 de julio. La variable independiente era el sexo y los dependientes eran: edad, procedencia, informante, tratamiento psiquiátrico previo, diagnóstico, medicamentos recetados y conducta. Resultados: de los 152 registros médicos analizados, se observa que: la edad predominante es de 60 a 69 años, la mayoría de ellos son mujeres, desde el municipio local y atendido a la atención de un acompañante. Los diagnósticos prevalentes son la esquizofrenia y los trastornos mentales y conductuales resultantes del uso de sustancias psicoactivas. Los medicamentos recetados con mayor frecuencia son la clase antipsicótica y anti-histamina, seguida de ansiolíticos/ sedantes. El criterio de Beers considera que la prescripción de fármacos del grupo de benzodiazepinas, antipsicóticos y antihistaminas, es inadecuada para los ancianos. La conducta principal era alta, sin remisión a otro servicio. Conclusión: aunque hay aliento del Ministerio de salud con la creación de nuevas políticas de salud mental, muchos profesionales mantienen el modelo de cuidado basado en la queja y la conducta, obstaculizando la rehabilitación psicosocial de los pacientes.

Descriptores: Ancianos; Servicios Psiquiátricos de Emergencia; Medicamentos; Registros Médicos. 


\section{Introdução}

O aumento da expectativa de vida e a mudança do perfil epidemiológico da população brasileira têm despertado, cada vez mais, o interesse dos pesquisadores acerca do envelhecimento, levando à necessidade da utilização de indicadores que ampliem a avaliação de saúde para o segmento da população idosa(1).

Já é sabido que o envelhecimento contribui para desencadear ou agravar a presença de transtornos mentais, tais como esquizofrenia, depressão, transtorno bipolar, delirante, de ansiedade, somatoforme, entre outros ${ }^{(2)}$. Um estudo europeu estimou que um em cada dois idosos havia experimentado um transtorno mental durante a vida e um em cada quatro apresentava-se afetado por doença mental no momento da entrevista. Esses autores reafirmaram a necessidade de disponibilização de serviços em saúde mental de boa qualidade para essa faixa etária(3).

A Portaria do Ministério da Saúde MS/GM n² 2528, de 19 de outubro de 2006, que aprovou a Política Nacional de Saúde da Pessoa Idosa, remete à promoção do envelhecimento ativo e saudável. Entre suas diretrizes, está descrita a necessidade de promoção de ações grupais integradoras e inclusivas, com avaliação, diagnóstico e tratamento da saúde mental da pessoa idosa(4). Nesse sentido, a Associação Brasileira de Psiquiatria propôs diretrizes para um modelo de atenção integral em saúde mental em nível terciário para atendimento de emergências psiquiátricas ${ }^{(5)}$.

$O$ atendimento de urgência e emergência psiquiátrica objetiva assistir o idoso em situações agudas, decorrentes ou não de doenças crônicas e progressivas, que representam a maior necessidade de cuidados para esta faixa etária(6). Esse serviço de urgência é utilizado pela população idosa em função da presença de fatores de risco e da pouca resolutividade dos outros setores da saúde no atendimento dessa população(7).

Frente ao exposto, conhecer as características de idosos que necessitam de atendimento em Pronto Socorro de Psiquiatria poderá contribuir com informações que subsidiem a melhor compreensão dessa realidade. Isto poderá desencadear novas reflexões e propostas de intervenções de melhoria do atendimento a essa especificidade, haja vista a existência de lacunas de estudos, especialmente epidemiológicos, sobre o tema.

Este trabalho teve por objetivo caracterizar os idosos atendidos em um Serviço de Urgência e Emergência Psiquiátrica em relação ao perfil sociodemográfico, diagnóstico psiquiátrico, prescrição de medicamentos e conduta médica e avaliar suas associações com o sexo.

\section{Método}

Trata-se de um estudo retrospectivo, analítico, baseado em dados secundários dos atendimentos prestados a idosos (60 anos ou mais) no Serviço de Urgência e Emergência Psiquiátrica de um hospital público de um município do interior do Estado de São Paulo, no período de 01/07/2015 a 30/06/2016. Em relação ao atendimento para a saúde mental, o referido município conta com uma unidade de internação psiquiátrica em hospital geral com 12 leitos, dois Centros de Atenção Psicossocial (CAPS), sendo um para tratamento de pessoas com transtornos mentais de longa evolução e outro para pessoas com transtornos mentais decorrentes do uso de substâncias psicoativas, 39 Unidades de Saúde da Família, 12 Unidades Básicas de Saúde, um hospital psiquiátrico, residências terapêuticas, um Ambulatório de Saúde Mental e uma oficina terapêutica.

O local do estudo constitui-se em um serviço de atendimento de urgência e emergência de uma unidade hospitalar de assistência e ensino que integra a rede de atenção à saúde do Departamento Regional de Saúde (DRS), cuja área de abrangência inclui 62 municípios, agrupados em cinco microrregiões, totalizando uma população estimada em um milhão e 200 mil habitantes.

O hospital constitui-se em cenário de ensinoaprendizagem para os cursos de Medicina e Enfermagem, além de disponibilizar campo de estágio para vários outros cursos da área da saúde de níveis superior e técnico, contribuindo, assim, com a formação de profissionais para o Sistema Único de Saúde (SUS).

Esse serviço de saúde, referência para a atenção especializada nos diferentes níveis de complexidade, tem, em sua estrutura, uma Unidade de Urgência e Emergência, constituindo-se como porta de entrada para as urgências/ emergências clínicas, cirúrgicas, psiquiátricas, ortopédicas e neurocirúrgicas, dentre outras.

O tamanho de amostra foi calculado utilizando-se os seguintes parâmetros estatísticos: população idosa atendida no serviço de Urgência e Emergência, entre 01/07/15 e 30/06/16 = 258; erro amostral de 5\%; nível de confiança de $95 \%$ e percentual máximo de $50 \%$, necessitando-se de 152 indivíduos. Foram incluídos os prontuários deles respeitando-se a proporção mensal dos atendimentos, o que garantiu que todos os meses do ano fossem contemplados. Prontuários com ausência de informações foram excluídos.

Os dados foram coletados no período de janeiro a março de 2017, obtidos por meio de coleta manual dos dados do prontuário dos pacientes, sendo todos usuários do SUS. Estes prontuários foram fornecidos pelo Setor de Prontuários do Complexo Assistencial da Instituição, nos quais foi possível obter as seguintes informações: idade; sexo; procedência; acompanhante ou informante; crise ou tratamento psiquiátrico anterior; hipótese diagnóstica de transtorno mental; medicamentos prescritos e conduta. Os dados relacionados à idade foram agrupados em faixas etárias de 60-69 anos, 70-79, 80 anos ou mais. Para 
a descrição das categorias dos fármacos, foi utilizada a classificação anatômica e terapêutica da Anatomical Therapeutic Chemical $(A T C)^{(8)}$. Os medicamentos potencialmente inapropriados (MPI) para idosos foram caracterizados de acordo com a atual revisão dos critérios de Beers, que identificam 53 medicamentos ou classes inadequadas para o uso em idosos, independentemente de diagnósticos e condições ${ }^{(9)}$.

No período do estudo, algum paciente pode ter utilizado o serviço supracitado mais de uma vez e, portanto, o número de atendimentos analisados pode superar o número de pacientes atendidos. Neste estudo, foi considerado o número de atendimentos, exceto para as características sociodemográficas, diagnósticos e antecedentes psiquiátricos.

Definiu-se o sexo como variável independente. As demais foram tratadas como variáveis dependentes. As análises estatísticas foram realizadas com o software SPSS, versão 17.0. As análises inferenciais foram realizadas por meio do teste de qui-quadrado de Pearson e a extensão do teste Exato de Fisher. Em todas as conclusões obtidas pelas análises inferenciais, utilizou-se o nível de significância a igual a 5\% ( $\leq \leq 0,05)$.

Esta pesquisa foi iniciada após aprovação pelo Comitê de Ética em Pesquisa para Seres Humanos da Faculdade de Medicina sob número CAAE 60175716.5.0000.5413, obtendo autorização do gestor hospitalar e dispensa do Termo de Consentimento Livre e Esclarecido (TCLE).

\section{Resultados}

No período referente à coleta de dados, foram obtidos os 152 prontuários e, neles, havia o registro de 321 atendimentos, tendo sido coletados dados do último atendimento registrado no mesmo.

Dos 152 prontuários analisados, foi possível observar que $53,3 \%$ dos pacientes eram do sexo feminino. A faixa etária entre 60 e 69 anos predominou nos dois sexos, bem como o local de procedência. A análise estatística mostra diferença significativa entre o sexo e a fonte de informação, sendo que os homens fornecem as próprias informações enquanto as informações das mulheres são fornecidas pelos acompanhantes. Além disso, mulheres apresentaram predomínio de somente um atendimento no período analisado (Tabela 1 ).

Tabela 1 - Caracterização dos idosos atendidos em um Serviço de Urgência e Emergência Psiquiátrica de um hospital público de acordo com sexo. Marília, SP, Brasil, 2015-2016

\begin{tabular}{lccccc}
\hline \multirow{2}{*}{ Variáveis } & \multicolumn{3}{c}{ Masculino (71) } & \multicolumn{3}{c}{ Feminino (81) } & $\mathbf{P}$ \\
\cline { 2 - 7 } & $\mathbf{N}$ & $\%$ & $\mathbf{n}$ & $\%$ & \\
\hline Idade (anos) & & & & & \\
$60-69$ & 43 & 60,0 & 50 & 61,7 & 0,88 \\
$70-79$ & 18 & 25,4 & 20 & 24,7 & 0,71 \\
\hline \multicolumn{1}{l}{} & & & & & (continua...)
\end{tabular}

\begin{tabular}{|c|c|c|c|c|c|}
\hline 80 ou + & 10 & 14,1 & 11 & 13,6 & 0,93 \\
\hline \multicolumn{6}{|l|}{ Procedência } \\
\hline Marília & 53 & 74,6 & 58 & 71,6 & 0,67 \\
\hline Outros municípios & 18 & 25,4 & 23 & 28,4 & \\
\hline \multicolumn{6}{|l|}{ Informante } \\
\hline Paciente & 40 & 56,3 & 32 & 39,5 & 0,04 \\
\hline Acompanhante & 30 & 42,2 & 48 & 59,2 & 0,04 \\
\hline $\begin{array}{l}\text { Não respondeu ao } \\
\text { chamado }\end{array}$ & 1 & 1,4 & 1 & 1,2 & 0,72 \\
\hline \multicolumn{6}{|l|}{$\mathrm{N}^{\circ}$ de atendimentos } \\
\hline Um & 45 & 63,4 & 64 & 79,0 & $0,03^{*}$ \\
\hline Dois & 12 & 16,9 & 7 & 8,6 & 0,12 \\
\hline Três & 7 & 9,9 & 3 & 3,7 & 0,11 \\
\hline Quatro & 1 & 1,4 & 2 & 2,5 & 0,55 \\
\hline Cinco ou mais & 6 & 8,5 & 5 & 6,2 & 0,41 \\
\hline
\end{tabular}

${ }^{*} \mathrm{p} \leq 0,05=$ Teste do qui-quadrado de Pearson

Em relação à quantidade de diagnósticos de transtorno mental, independentemente do sexo, observa-se predomínio de um único diagnóstico. Também foi possível observar que o diagnóstico de "Transtornos mentais e de comportamento decorrentes do uso de substâncias psicoativas" foi mais frequente em homens. Os demais diagnósticos de transtorno mental não mostraram diferenças significativas ao serem comparados em relação ao sexo. A alta foi a conduta mais presente após os atendimentos, seguida pela internação, em ambos os sexos (Tabela 2).

Tabela 2 - Diagnósticos de transtorno mental e conduta após consultas médicas de idosos atendidos em um Serviço de Urgência e Emergência Psiquiátrica de um hospital público de acordo com sexo. Marília, SP, Brasil, 2015-2016

\begin{tabular}{|c|c|c|c|c|c|}
\hline \multirow[t]{2}{*}{ Variáveis } & \multicolumn{2}{|c|}{ Masculino } & \multicolumn{2}{|c|}{ Feminino } & \multirow[t]{2}{*}{$p$} \\
\hline & $\mathbf{N}$ & $\%$ & $\mathbf{n}$ & $\%$ & \\
\hline \multicolumn{6}{|l|}{$\begin{array}{l}\mathrm{N}^{\circ} \text { de diagnósticos na } \\
\text { última consulta }\end{array}$} \\
\hline 1 Diagnóstico & 62 & 87,3 & 70 & 86,4 & 0,80 \\
\hline 2 diagnósticos & 2 & 2,8 & 3 & 3,7 & 0,56 \\
\hline 3 diagnósticos & 0 & 0 & 1 & 1,2 & 0,53 \\
\hline Nenhum diagnóstico & 6 & 8,5 & 4 & 4,9 & 0,29 \\
\hline $\begin{array}{l}\text { Não respondeu ao } \\
\text { chamado }\end{array}$ & 1 & 1,4 & 3 & 3,7 & 0,28 \\
\hline \multicolumn{6}{|l|}{ Diagnósticos } \\
\hline $\begin{array}{l}\text { Transtornos mentais } \\
\text { orgânicos }\end{array}$ & 15 & 22,6 & 12 & 17,1 & 0,43 \\
\hline $\begin{array}{l}\text { Transtornos mentais } \\
\text { e de comportamento } \\
\text { decorrentes do uso de } \\
\text { substância psicoativa }\end{array}$ & 24 & 37,1 & 5 & 7,1 & $0,001^{*}$ \\
\hline $\begin{array}{l}\text { Esquizofrenia, transtorno } \\
\text { esquizotípico e delirante }\end{array}$ & 11 & 17,7 & 22 & 31,4 & 0,07 \\
\hline $\begin{array}{l}\text { Transtornos de humor } \\
\text { (afetivos) }\end{array}$ & 7 & 11,3 & 13 & 18,6 & 0,24 \\
\hline $\begin{array}{l}\text { Transtornos neuróticos, } \\
\text { relacionados ao estresse } \\
\text { e somatoforme }\end{array}$ & 6 & 9,7 & 13 & 18,6 & 0,15 \\
\hline $\begin{array}{l}\text { Síndromes } \\
\text { comportamentais } \\
\text { associadas a } \\
\text { perturbações fisiológicas } \\
\text { e fatores físicos }\end{array}$ & 0 & 0 & 2 & 2,9 & 0,28 \\
\hline
\end{tabular}




\begin{tabular}{|c|c|c|c|c|c|}
\hline $\begin{array}{l}\text { Transtornos de } \\
\text { personalidade e de } \\
\text { comportamento }\end{array}$ & 1 & 1,6 & 3 & 4,3 & 0,36 \\
\hline \multicolumn{6}{|l|}{ Conduta } \\
\hline Alta & 36 & 49,3 & 44 & 54,3 & 0,90 \\
\hline Internação & 25 & 35,2 & 20 & 24,7 & 0,16 \\
\hline Revelia & 2 & 2,8 & 2 & 2,5 & 0,64 \\
\hline $\begin{array}{l}\text { Alta e encaminhamento - } \\
\text { CAPS AD }\end{array}$ & 3 & 4,2 & 1 & 1,2 & 0,47 \\
\hline $\begin{array}{l}\text { Alta e encaminhamento } \\
-\mathrm{ASM}^{\ddagger}\end{array}$ & 4 & 5,6 & 9 & 11,1 & 0,25 \\
\hline $\begin{array}{l}\text { Não respondeu ao } \\
\text { chamando }\end{array}$ & 1 & 2,8 & 2 & 2,5 & 0,45 \\
\hline Sem informação & 0 & 0 & 3 & 3,7 & 0,08 \\
\hline
\end{tabular}

${ }^{*} p \leq 0,05=$ Teste Exato de Fisher; ${ }^{+}$CAPS AD = Centro de Atenção Psicossocial Álcool e Drogas; ${ }^{\ddagger} \mathrm{ASM}=$ Ambulatório de Saúde Mental

Na Tabela 3, é possível observar que, dos 152 pacientes atendidos no serviço, 30 homens e 48 mulheres foram medicados e as mulheres receberam mais medicamento que os homens $(p=0,04)$. Os dados também mostraram o predomínio do uso de um medicamento prescrito, independentemente do sexo do paciente. A Tabela 3 demonstra, ainda, as classes dos medicamentos prescritos no momento dos atendimentos médicos. É possível observar que prevalece a associação entre um antipsicótico e um anti-histamínico, predominando o uso de haloperidol e prometazina. O segundo maior grupo de medicamentos presente nas prescrições foi o dos ansiolíticos/sedativos, predominando a prescrição de alprazolam. Ao se confrontar os medicamentos prescritos com os constantes no Critério de Beers ${ }^{(9)}$, nota-se a presença de alguns benzodiazepínicos, de um antipsicótico e de um anti-histamínico. A análise estatística mostrou que mulheres receberam mais prescrição de alprazolam ao serem comparadas com homens.

Tabela 3 - Perfil de uso e classe de medicamentos prescritos para idosos atendidos em um Serviço de Urgência e Emergência Psiquiátrica de um hospital de acordo com sexo. Marília, SP, Brasil, 2015-2016

\begin{tabular}{|c|c|c|c|c|c|}
\hline \multirow[t]{2}{*}{ Variáveis } & \multicolumn{2}{|c|}{ Masculino } & \multicolumn{2}{|c|}{ Feminino } & \multirow[t]{2}{*}{$\mathbf{P}$} \\
\hline & $\mathbf{n}$ & $\%$ & $\mathbf{n}$ & $\%$ & \\
\hline \multicolumn{6}{|l|}{ Recebeu medicação } \\
\hline Sim & 30 & 42,3 & 48 & 59,3 & $0,04^{*}$ \\
\hline Não & 37 & 52,1 & 29 & 35,8 & $0,04^{*}$ \\
\hline Não respondeu ao chamado & 4 & 5,6 & 4 & 4,9 & 0,56 \\
\hline \multicolumn{6}{|l|}{$\begin{array}{l}\text { Número de medicamentos } \\
\text { prescritos }\end{array}$} \\
\hline 1 medicamento & 13 & 4,3 & 28 & 58,3 & 0,20 \\
\hline 2 medicamentos & 17 & 56,7 & 19 & 39,6 & 0,14 \\
\hline 3 ou mais medicamentos & 0 & 0 & 1 & 2,1 & 0,61 \\
\hline \multicolumn{6}{|l|}{ Medicamento } \\
\hline Antipsicótico típico & 5 & 16,7 & 9 & 18,7 & 0,53 \\
\hline Antipsicótico atípico & 1 & 3,3 & 2 & 4,2 & 0,67 \\
\hline Ansiolítico/sedativo & 6 & 20,0 & 17 & 35,4 & 0,15 \\
\hline Anticolinérgico & 1 & 3,3 & 0 & 0 & 0,38 \\
\hline $\begin{array}{l}\text { Antipsicótico típico + } \\
\text { ansiolítico }\end{array}$ & 2 & 6,7 & 1 & 2,1 & 0,32 \\
\hline
\end{tabular}

\begin{tabular}{lccccc} 
(Tabela 3 - continuação) & \multicolumn{7}{c}{ Masculino } & \multicolumn{2}{c}{ Feminino } & $\mathbf{P}$ \\
\hline Variáveis & $\mathbf{n}$ & $\%$ & $\mathbf{n}$ & $\%$ & \\
\hline $\begin{array}{l}\text { Antipsicótico típico + anti- } \\
\text { histamínico }\end{array}$ & 14 & 46,7 & 18 & 37,5 & 0,42 \\
$\quad \begin{array}{l}\text { Antipsicótico atípico + anti- } \\
\text { histamínico }\end{array}$ & 1 & 3,3 & 0 & 0 & 0,38 \\
2 antipsicóticos típicos + anti- & 0 & 0 & 1 & 2,1 & 0,61 \\
$\quad$ histamínicos & & & & & \\
Medicamentos & & & & & \\
potencialmente inapropriados & & & & & \\
(Critério de Beers) & & & & & \\
$\quad$ Alprazolam & 1 & 4,2 & 9 & 25,0 & $0,03^{\dagger}$ \\
$\quad$ Clonazepam & 2 & 8,3 & 5 & 13,9 & 0,41 \\
$\quad$ Diazepam & 1 & 4,2 & 2 & 5,6 & 0,65 \\
$\quad$ Lorazepam & 2 & 8,3 & 0 & 0 & 0,16 \\
$\quad$ Clorpromazina & 3 & 12,5 & 1 & 2,8 & 0,17 \\
$\quad$ Prometazina & 15 & 62,5 & 19 & 52,8 & 0,46 \\
\hline
\end{tabular}

${ }^{*} p \leq 0,05=$ Teste do qui-quadrado de Pearson; ${ }^{+} p \leq 0,05=$ Teste Exato de Fisher

\section{Discussão}

Ao se comparar os dados encontrados nos prontuários analisados neste estudo com dados da literatura, observa-se que há semelhança em diversos aspectos.

Quanto ao sexo, a maior presença feminina pode ser justificada pelo papel historicamente desempenhado pela mulher na sociedade, a qual assume múltiplas obrigações e responsabilidades, como a de cuidar dos filhos, associadas à inserção no mercado de trabalho, podendo favorecer o surgimento das doenças mentais, como, por exemplo, a depressão(10).

Em estudo que estabeleceu o perfil sociodemográfico de pacientes em um Centro de Atenção Psicossocial, foi apontado que, de uma maneira geral, as mulheres buscam mais os serviços de saúde do que os homens. Aspectos como o autocuidado, mais presente na população feminina, e o receio do homem em encontrar aspectos negativos em relação à sua saúde mental são fatores que podem diminuir a procura dos homens pelos serviços de saúde ${ }^{(11)}$.

No que se refere à idade, a prevalência maior de atendimentos foi identificada na faixa etária entre 60 e 69 anos. Estudo realizado em um centro que atende exclusivamente transtornos mentais, foi verificada uma prevalência de $60,4 \%$ na faixa etária dos 60 aos 69 anos ${ }^{(12)}$. Não foram encontradas, na literatura, justificativas para essa faixa etária ser a mais acometida por transtornos mentais, mas se pode entender que é o início da velhice, fase de maior risco para diversas doenças, o que pode justificar a maior procura nos Serviços de Urgência e Emergência Psiquiátrica.

Quanto à procedência dos atendidos, 73\% foram do município em que foi realizado o estudo, embora o local seja referência para 62 municípios. Segundo o IBGE ${ }^{(13)}$, esse município tem uma população estimada em 216.745 habitantes, o que pode justificar o maior número de atendimentos de idosos oriundos do mesmo. 
Quanto à fonte de informações no ato do atendimento, homens responsabilizaram-se mais que mulheres por isso. É reconhecido que a necessidade do homem idoso em se manter independente e autônomo está sustentada na concepção de masculinidade. Esse comportamento, de um lado, pode ser benéfico ao se considerar o autocuidado e a responsabilidade pela própria saúde e, de outro, pode expressar a negação de problemas de saúde, tornando-se um risco de agravamento de condições de saúde. Em contrapartida, mulheres idosas salientam a importância das relações de amparo e proteção da família e da manutenção da saúde ${ }^{(14)}$. Dados da literatura demonstram que os aspectos anteriormente apresentados passam a ser centrais nas vidas dos idosos, principalmente para as mulheres que identificaram, nessa etapa, perdas relativas aos laços sociais familiares ${ }^{(15)}$.

Neste estudo, destacam-se os quadros de transtornos mentais e comportamentais decorrentes do uso de substâncias psicoativas $(17,8 \%)$, seguidos por esquizofrenia (15,8\%). Dados da literatura mostram que os diagnósticos de maior incidência, em ambos os sexos, foram os transtornos do humor - episódio depressivo, com $14,2 \%$, seguido por esquizofrenia, com $11,8 \%$, e os transtornos mentais e comportamentais decorrentes do uso do álcool representaram 9,3\% dos $\operatorname{casos}^{(12)}$. O uso de substâncias psicoativas como o álcool é considerado ato social e está presente em diferentes culturas nas quais os indivíduos buscam desinibição, relaxamento e, inclusive, efeitos afrodisíacos ${ }^{(16)}$. O consumo de álcool e de outros elementos psicoativos por pessoas idosas ocasiona impactos significativos nas condições de saúde e efeitos importantes no bem-estar e qualidade de vida. O uso dessas substâncias, muitas vezes, é deflagrado a partir de situações de vivências pelos idosos, como é o caso de perdas, limitações físicas e a própria aposentadoria. Além disso, as relações construídas ao longo da vida pelos idosos e o consumo de álcool e outras drogas representam um fenômeno complexo(17).

A esquizofrenia é o diagnóstico bastante presente nos prontuários analisados. Essa doença afeta aproximadamente $1 \%$ da população mundial, sendo que, no Brasil, entre 2008 e 2014, houve 51.640 internações de idosos devido à esquizofrenia(18). Compatível com o diagnóstico de esquizofrenia está a presença da prescrição de antipsicóticos, tanto típicos quanto atípicos, em ambos os sexos.

Quanto à conduta médica no atendimento, a mais frequente neste estudo foi a com $52 \%$, seguida de internação, com 29,6\%. Já a maioria dos idosos atendidos no Serviço de Emergência Psiquiátrica do Centro de Atenção Integrada à Saúde Mental (UEP-CAISM) foi encaminhada para o ambulatório(12). Em outro estudo, realizado em um serviço de emergência em Sobral-CE, $43,45 \%$ dos pacientes foram encaminhados ao CAPS-ad local $^{(19)}$, ou seja, parte significativa dos pacientes foi encaminhada a um serviço que integra o novo modelo de atenção em saúde mental. Este novo modelo surgiu como proposta da Rede de Atenção Psicossocial, que foi instituída em 23 de dezembro de 2011, pela Portaria no 3.088 , e tem como finalidade garantir a integralidade do cuidado, garantindo a articulação e integração das redes, qualificando $o$ atendimento. Os serviços disponíveis dentro desta rede são: Atenção Básica em Saúde (ESF, NASF); Atenção Psicossocial Especializada (CAPS); Atenção Residencial de Caráter Transitório; Urgência e Emergência; Atenção Hospitalar; Estratégia de Desinstitucionalização e Reabilitação Psicossocial(20).

Dos 152 prontuários de pacientes analisados, 51,3\% receberam medicação, sendo que, dentre estes, 52,3\% receberam apenas um medicamento. Vale ressaltar que, embora a análise estatística não tenha mostrado diferença significativa, homens apresentaram predomínio de prescrição de dois medicamentos enquanto mulheres, de apenas um. Ao se relacionar o número de medicamentos prescritos com a classe de medicamentos, a Tabela 3 mostra o predomínio da prescrição da associação de antipsicótico com anti-histamínico para homens, o que poderia justificar a maior prescrição de dois medicamentos para homens. Já em relação às mulheres, observa-se tanto a prescrição de ansiolítico/sedativo quanto de antipsicótico associado a anti-histamínico, não permitindo relacionar diretamente a quantidade de medicamentos com as respectivas classes farmacológicas.

Em relação ao uso dos psicofármacos prescritos, a literatura descreve os principais medicamentos preconizados na rede pública. De acordo com a Relação Nacional de Medicamentos Essenciais - RENAME(21), os medicamentos como alprazolam, bromazepam e lorazepam, que foram prescritos, não constam em sua listagem de medicamentos essenciais. A RENAME foi criada como proposta pela Organização Mundial de Saúde (OMS) como forma de selecionar os medicamentos essenciais conforme o custo acessível, a qualidade e segurança e ainda de acordo com sua eficácia e relevância para a saúde da população(22). A prescrição de medicamentos por um serviço público não constantes na RENAME pode implicar a não adesão ao tratamento, uma vez que o usuário terá que adquirir o mesmo em farmácias comerciais.

Ainda em relação aos medicamentos que foram classificados como potencialmente inapropriados para idosos, de acordo com o Critério de Beers ${ }^{(9)}$, a maioria é representada pela classe dos benzodiazepínicos. Dados da literatura mostram que o consumo de drogas que atuam no sistema nervoso central, principalmente os benzodiazepínicos, é muito alto(23). Esse grupo de medicamentos é indicado para o manejo de doenças psiquiátricas, tais como ansiedade, alguns tipos de convulsão e como relaxantes musculares, 
anticonvulsivantes, tendo sua posologia prescrita por curto tempo, não devendo ultrapassar três meses ${ }^{(9,24)}$. Esses medicamentos podem ocasionar sintomas como sonolência diurna, redução da coordenação motora, alteração da memória, tontura, zumbidos, delirium, quedas e fraturas, reação paradoxal, intoxicação, além do risco de dependência. O uso em longo prazo, em idosos, parece estar relacionado ao aumento do risco de demência, principalmente da doença de Alzheimer ${ }^{(9,23-24)}$. Além disso, sua ação depressora no sistema nervoso central, quando associada aos outros medicamentos, o que é relativamente comum no idoso, pode ter seu efeito sedativo potencializado e até inibir o centro respiratório bulbar, levando a uma insuficiência respiratória(25).

Nesse sentido, embora os benzodiazepínicos sejam amplamente utilizados em idosos, não se recomenda o seu uso pelo fato de que, com o avanço da idade, há alterações qualitativas e quantitativas no complexo GABAérgico, particularmente em relação aos receptores GABA, que são responsáveis pelo aumento da sensibilidade aos benzodiazepínicos ${ }^{(26)}$. Assim, para o uso de benzodiazepínicos em idosos, faz-se necessária uma adequação da dose, reduzindo-a, para alcançar o mesmo nível de sedação atingido em jovens. Ainda em relação aos idosos, a prescrição de benzodiazepínicos só deve ocorrer quando for extremamente necessária, dando-se sempre preferência aos fármacos de meia-vida curta e intermediária(27).

Apesar da importância deste estudo, deve-se levar em conta a limitação do mesmo, visto que não é possível a extrapolação dos dados obtidos para toda população de idosos, uma vez que os dados foram provenientes de um serviço público de atenção à saúde. Porém, apesar das limitações, este estudo evidenciou importantes características acerca da prescrição de medicamentos para idosos em Serviço de Urgência e Emergência Psiquiátrica.

\section{Conclusão}

A caracterização dos idosos atendidos no local do estudo mostrou que a idade mais prevalente é de 60 a 69 anos, a maioria é composta por mulheres, procedentes do município local e que compareceram ao atendimento com acompanhante. Dos 152 idosos, 132 receberam apenas um diagnóstico, sendo os mais prevalentes a esquizofrenia e transtornos mentais e comportamentais decorrentes do uso de substâncias psicoativas. A principal conduta foi a alta. Ao serem atendidos, receberam prescrição de medicamentos, na maioria, apenas um, sendo a classe do ansiolítico/sedativo a mais frequente. O critério de Beers identificou a prescrição de medicamentos do grupo dos benzodiazepínicos, antipsicóticos e anti-histamínicos, os quais são considerados medicamentos inapropriados para idosos.
Esses resultados sugerem que o atendimento realizado não ocorre com base no modelo de clínica ampliada e da Política Nacional de Humanização, uma vez que, dentre as condutas mais frequentes, estão a alta sem encaminhamento e a internação, sendo possível inferir que, no atendimento, houve valorização somente da queixa atual do paciente, sem a preocupação de inseri-lo nos demais serviços de saúde mental que compõem a Rede de Atenção Psicossocial. Tal fato dificulta a implantação de um novo modelo de atenção em saúde mental que atenda os pacientes em suas diversas necessidades de saúde e contribua para a sua reabilitação psicossocial, oferecendo-lhes opções de tratamentos que possibilitem a volta às suas rotinas, incluindo-os no mercado de trabalho e garantindo o tratamento necessário.

\section{Referências}

1. Miranda GMDM, Mendes ACG, Silva ALA. O envelhecimento populacional brasileiro: desafios e consequências sociais atuais e futuras. Rev Bras Geriatr Gerontol. [Internet] 2016;19(3):507-519. [cited jan 29 2019]. Available from:<http://www.scielo.br/scielo. php?pid=S1809-98232016000300507\&script $=$ sci_ arttext\&tlng=pt.>

2. Tavares SMG. A saúde mental do idoso brasileiro e sua autonomia. BIS Bol Inst Saude. 2009;47:87-9.

3. Andreas S, Schulz H, Volkert J, Dehoust M, Sehner $S$, Suling $A$, et al. Prevalence of mental disorders in elderly people: the European MentDis_ICF65+ study. Br J Psychiatry. 2017;210:125-31. doi: 10.1192/bjp. bp. 115.180463.

4. Brasil. Ministério de Saúde. Portaria n. 2528/GM, de 19 de outubro de 2006. Aprova a Política Nacional de Saúde da Pessoa Idosa [Internet]. Brasília; 2006 [cited jan 22 2019]. Available from: http://bvsms.saude.gov.br/bvs/ saudelegis/gm/2006/prt2528_19_10_2006.html

5. Associação Brasileira de Psiquiatria et al. Diretrizes para um modelo de assistência integral em saúde mental no Brasil. Rio de Janeiro: ABP, 2014. [cited jan 20 2019]. Available from: <http://www.abpbrasil.org.br/diretrizes_ final.pdf $>$.

6. Yonekura T, Quintans JR, Kato MY, Filho AN. A atenção à saúde do idoso nos serviços brasileiros de urgência: uma revisão integrativa. Sau. Transf. Soc. 2016;6(2):97-113. 7. Pilger C, Menon MU, Mathias TAF. Utilização de serviços de saúde por idosos vivendo na comunidade. Rev Esc Enferm USP. 2013;47(1):213-20.

8. World Health Organization. Anatomical tharapeutic chemical - ATC classification index with defined daily doses - DDD's. Olso; 2018.

9. American Geriatrics Society. American Geriatrics Society 2015 updated beers criteria for potentially inappropriate medication use in older adults. J Am Geriatr Soc. 2015;63(11):2227-46. 
10. Carneiro AC, Murmel JG, Werner R. Relações de gênero e saúde mental: a marginalização da mulher com transtorno mental. In: FÓRUM DE DIREITOS HUMANOS E SAÚDE MENTAL, 3. 2017. Anais eletrônicos... Florianópolis: ABRASME, 2017. [Internet] 2017 [cited jan 27 2019]. Available from: <http://www.direitoshumanos2017. abrasme.org.br/resources/anais/8/1489545418_ ARQUIVO_Artigoabrasme.pdf>. Acesso em: 20 ago. 2017. 11. Campos IO, Ramalho WM, Zanello V. Saúde mental e gênero: o perfil sociodemográfico de pacientes em um centro de atenção psicossocial. Estudos de Psicologia. 2017;22(1):68-77. doi: 10.22491/1678-4669.20170008 12. Baldaçara L, Batista IAGL, Neves AAM, Silva I, Jackowski AP. Emergências psiquiátricas nos idosos. Estudo epidemiológico. Arq Méd Hosp Fac Ciênc Méd Santa Casa São Paulo. [Internet]. 2012;57(1):11-18. [Cited jan 28 2019] Available from: <http://www.fcmsantacasasp.edu. br/images/Arquivos_medicos/2012/57_1/03-Artigo\%20 Original\%2032.pdf>.

13. Brasil. Ministério do Planejamento, Orçamento e Gestão. Instituto Brasileiro de Geografia e Estatística. Contagem Populacional. [Cited jan 28 2019] Available from: <https://cidades.ibge.gov.br/painel/painel. php?codmun $=352900>$.

14. Coelho JS, Giacomin KC, Firmo JOA. O cuidado em saúde na velhice: a visão do homem. Saúde Soc. [Internet]. 2016;25(2):408-421. http://dx.doi. org/10.1590/S0104-12902016142920.

15. Camargo BV, Contarello A, Wachelke JFR, Morais DX, Piccolo $C$. Representações sociais do envelhecimento entre diferentes gerações no Brasil e na Itália. Psicologia em Pesquisa. 2014;8(2):179-188.

16. Silva ECS, Oliveira JAP. Dependência do álcool na terceira idade: causas, consequências e desafios para a família e profissionais da área da psicologia. Rev Psicol Saúde e Debate. [Internet] 2018;4(3):46-59. [Cited fev 06 2019]. Available from: https://www.psicodebate. dpgpsifpm.com.br/index.php/periodico/article/view/ V4N3A5/256

17. Ottoni MAM. Longevidade: uma conquista ou um peso para a sociedade brasileira?. Polêmica, [Internet]. 2014;SI,13(1):996-1005. [Cited fev 05 2019]. Available from: <https://www.e-publicacoes.uerj.br/index.php/ polemica/article/view/9630/7556>.

18 Santos VC, Anjos KF, Boery RNSO, Moreira RM, Cruz DP, Boery EN. Internação e mortalidade hospitalar de idosos por transtornos mentais e comportamentais no Brasil, 2008-2014. Epidemiol Serv Saúde. [Internet]. 2017;26(1):39-49. [Cited fev 05 2019]. Available from: http://www.scielo.br/scielo.php?pid=S223796222017000100039\&script=sci_abstract\&tlng=pt. 19 Sousa FSP, Silva CAF, Oliveira EM. Serviço de Emergência Psiquiátrica em hospital geral: estudo retrospectivo. Rev
Esc Enferm USP. 2010;44(3):796-802. doi: http://dx.doi. org/10.1590/S0080-62342010000300035.

20. Brasil. Ministério da Saúde. Portaria n 3088, de 23 de dezembro de 2011. Institui a Rede de Atenção Psicossocial para pessoas com sofrimento ou transtorno mental e com necessidades decorrentes do uso de crack, álcool e outras drogas, no âmbito do Sistema Único de Saúde (SUS). [Internet]. Brasília; 2011 [cited fev 11 2019]. Available from:http://bvsms.saude.gov.br/bvs/saudelegis/ gm/2011/prt3088_23_12_2011_rep.html

21. Brasil. Ministério da Saúde. Secretaria de Ciência, Tecnologia e Insumos Estratégicos. Departamento de Assistência Farmacêutica e Insumos Estratégicos. Relação Nacional de Medicamentos Essenciais: RENAME 2014. - 9. ed. rev. e atual. - Brasília: Ministério da Saúde, 2015. 22. Brasil. Ministério da Saúde. Secretaria de Políticas de Saúde. Departamento de Atenção Básica. Política nacional de medicamentos 2001. Brasília: Ministério da Saúde, 2001.

23. Manso, MEG, Biffi ECA, Gerardi TJ. Prescrição inadequada de medicamentos a idosos portadores de doenças crônicas em um plano de saúde no município de São Paulo, Brasil. Rev Bras Geriat Geront. 2015;18(1):151-164.

24. LopesLM, Figueiredo TP, Costa SC, Reis AMM. Utilização de medicamentos potencialmente inapropriados por idosos em domicílio. Ciência Saúde Coletiva. [Internet] 2016;21(11):3429-3438. [Cited fev 11 2019]. Available from: http://www.scielo.br/scielo.php?pid=S141381232016001103429\&script=sci_abstract\&tIng=pt 25. Viel AM, Ribeiro-Paes JT, Stessuk T, Santos L. Interações medicamentosas potenciais com benzodiazepínicos em prescrições médicas de pacientes hospitalizados. Revista de Ciências Farmacêuticas Básica e Aplicada. 2014;35(4);589-596.

26. Turnheim K. Drug therapy in the elderly. Exp Gerontol. 2004;39(11-12):1731-8

27. Andrade KVF, Silva Filho C, Junqueira LL. Prescrição de medicamentos potencialmente inapropriados para idosos: um estudo transversal em instituição psiquiátrica. J Bras Psiquiatr. 2016;65(2):149-54. doi: 10.1590/0047-2085000000116

\section{Contribuição dos Autores}

Concepção e planejamento do estudo: Mayara Giuli dos Santos Cordeiro, Maria José Sanches Marin, Carlos Alberto Lazarini. Obtenção dos dados: Mayara Giuli dos Santos Cordeiro, Maria José Sanches Marin, Carlos Alberto Lazarini. Análise e interpretação dos dados: Mayara Giuli dos Santos Cordeiro, Márcia Aparecida Padovan Otani, Flávia Cristina Goulart, Osni Lazaro Pinheiro, Maria José Sanches Marin, Carlos Alberto Lazarini. Análise estatística: Mayara Giuli dos Santos Cordeiro, Márcia Aparecida Padovan Otani, Flávia Cristina 
Goulart, Osni Lazaro Pinheiro, Maria José Sanches Marin, Carlos Alberto Lazarini. Redação do manuscrito: Mayara Giuli dos Santos Cordeiro, Márcia Aparecida Padovan Otani, Flávia Cristina Goulart, Osni Lazaro Pinheiro, Maria José Sanches Marin, Carlos Alberto Lazarini. Revisão crítica do manuscrito: Mayara Giuli dos Santos Cordeiro, Márcia Aparecida Padovan Otani, Flávia Cristina Goulart, Osni Lazaro Pinheiro, Maria José Sanches Marin, Carlos Alberto Lazarini.

Todos os autores aprovaram a versão final do texto. Conflito de interesse: os autores declararam que não há conflito de interesse. 